\title{
Performance based Channel Allocation in IEEE 802.22 Networks
}

\author{
Saptarshi Debroy Shameek Bhattacharjee and Mainak Chatterjee \\ Department of Electrical Engineering \& Computer Science \\ University of Central Florida \\ Orlando, FL 32816 \\ Email: \{saptarsh, shameek, mainak\}@eecs.ucf.edu
}

\begin{abstract}
The main challenge in resource allocation in cognitive radio based IEEE 802.22 networks is the absence of predefined control channels. Moreover, the fleeting nature of the available spectrum also hinders the communication as the radios must relinquish the acquired channels once the primary users of those channels return.

In this paper, we propose a performance metrics based data channel allocation scheme for IEEE 802.22 networks where the base station allocates interference free channels to the consumer premise equipments using a spectrum map. The base station creates the spectrum map by using the raw spectrum usage data that are shared by a small subset of consumer premise equipments. The usage data are fused at the base station using a modified version of Shepard's interpolation technique. We construct a continuous and differentiable spatial distribution of spectrum usage that the base station consults to estimate the spectrum occupancy vector at any arbitrary location in its cell. Such spectrum usage is then utilized to proactively evaluate some key network and radio performance metrics which in turn help allocating the best candidate channel to a given consumer premise equipment ensuring highest achievable performance.
\end{abstract}

\section{INTRODUCTION}

The IEEE 802.22 wireless regional area network (WRAN) [2] is a cognitive radio based technology [3] that exploits the unused sub- $900 \mathrm{MHz}$ bands. These bands are primarily owned and licensed by TV stations (primary users). The core components of an IEEE 802.22 network, base stations (BS) and the consumer premise equipments (CPE), continuously scan the entire spectrum and opportunistically access the unused or underutilized TV bands. Channel allocation to the CPEs are done by the BS. Upon detection of any transmission activity from a primary user on the channels being used, the secondary users are mandated to relinquish those channels [1]. The ability of the BS to assign the best possible channels to the CPEs depends on how well the $\mathrm{BS}$ is able to capture the spectrum availability at the various CPE locations. Since the BS cannot perform sensing at locations other than itself, it has to rely upon the sensed spectrum reports shared by the CPEs. If all the CPEs were to continuously share their spectrum usage reports, the BS would have the most accurate information. However, the communication overhead becomes a bottleneck as sharing of data has to be done on the same channels that the BS is

This research was sponsored by National Science Foundation, under award no.CCF-0950342. supposed to allocate. Thus channel assignment to CPEs is a challenging problem.

Most of the existing work in IEEE 802.22 networks are focused on self-coexistence [12], signal detection or spectrum sensing [4], [13], inter-cell and control channel assignment [6]; however, to the best of our knowledge, no significant work has been done investigating channel allocation within an IEEE 802.22 cell.

In this paper, we propose an on-demand channel allocation scheme for IEEE 802.22 networks where the BSs allocate unused channels to CPEs. We argue that the lack of knowledge about the channel usage scenario at the CPE locations is the primary difficulty in efficient channel assignment. To counter this, we propose the creation and maintenance of a Spectrum Map at the BS. A Spectrum Map describes the spectrum usage of an area under the purview of a given BS. To create a Spectrum Map, the BS elects a subset of CPEs, called delegates, to opportunistically share their spectrum usage with the BS. The BS intelligently fuses such spectrum usage data and uses Shepard's interpolation [11] method to construct the Spectrum Map. The map is used for quicker communication with a candidate $\mathrm{CPE}$ and increases the probability of synchronization between the CPE and the BS. Such communication allows the BS to acquire the actual spectrum usage at the CPE and evaluate different performance metrics such as wireless capacity, secondary network throughput, spectrum efficiency, and bit error rate for candidate channels. Such proactive performance analysis not only identifies the best candidate channel for a CPE but also indicates the best possible CPE among candidate CPEs for a particular channel. Channel allocation scheme thus adopted increases the overall network throughput and maximizes channel performance.

To evaluate our scheme, we construct a realistic simulation environment emulating the location distribution of digital TV transmitters in US [8], [9] and duplicating their transmission patterns. We deploy IEEE 802.22 networks at different locations with varying population density of primaries. We compare the results with actual received signal strength values. Comparisons show a low probability of error $(p=0.06)$ for false positives and upto $92 \%$ reduction in redundant beacon broadcast which increases the probability of synchronization. Finally, the allocation algorithm shows close to perfect allocation and more than $50 \%$ of free channel utilization even in worst case scenario with no intra-cell spatial reuse. 


\section{SySTEM MODEL}

We consider an IEEE 802.22 network that is divided into cells, each having a BS. A BS can communicate with the CPEs in its cell as well as with its neighboring BSs.

We assume that all BSs continuously perform channel sensing, i.e., they scan the entire spectrum (from 54 to 698 $\mathrm{MHz}$ ) and create their respective spectrum usage report. The IEEE 802.22 draft [1] requires all the devices in the network to be installed in fixed locations and the BS to be aware of the location of all the CPEs under it. According to [1] there are no pre-defined control channels in the system and the only means of communication between the BS and the CPE are the free channels that are currently not being used by the primary users. Apart from allocating free channels to the CPE, the BS is also responsible for transmitting beacons to advertise free channels, gather sensed data from the CPEs, and fuse those spectrum data. We select a subset of CPEs that we call delegation CPEs. These delegated CPEs are chosen in a dynamic manner based on their location and transmission activity.

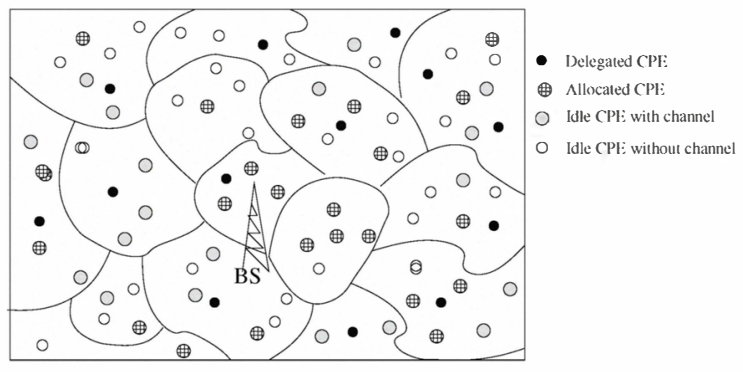

Fig. 1. IEEE 802.22 network with BS and different types of CPEs

\section{A. Zones}

Zones are logical divisions of the coverage area of BS. This division is proposed as a BS specific feature and therefore does not violate [1] specifications. The purpose of such zonal division is efficient channel allocation. Such division is governed by the requirement to keep similar densities of CPEs per zone. A CPE cannot be allocated any channel which is being used by the allocated or delegated CPEs of that zone unless they are beyond the interference region of the CPE. The zones are also important for selecting the delegation CPEs. The idea is to have almost equal representation from all zones and no zone is unrepresented as far as possible. The BS maintains three queues for each zone, namely: AQ (for allocated CPEs), DQ (for delegated CPEs) and HQ (for potentially delegate CPEs i.e., idle CPEs with channels).

\section{B. Delegation of CPEs}

The underlying principle of efficient channel allocation is the ability of the BS to successfully predict the spectrum usage at any CPE location in the cell. To achieve this, the BS needs to periodically collect raw spectrum usage data from the delegated CPEs. At any instance, only delegated CPEs take part in sharing their spectrum usage with the BS. A prudent choice of delegation CPEs results in better interpolation of spectrum usage. BS controls the selection of a particular CPE as a delegation member. BS aims to have

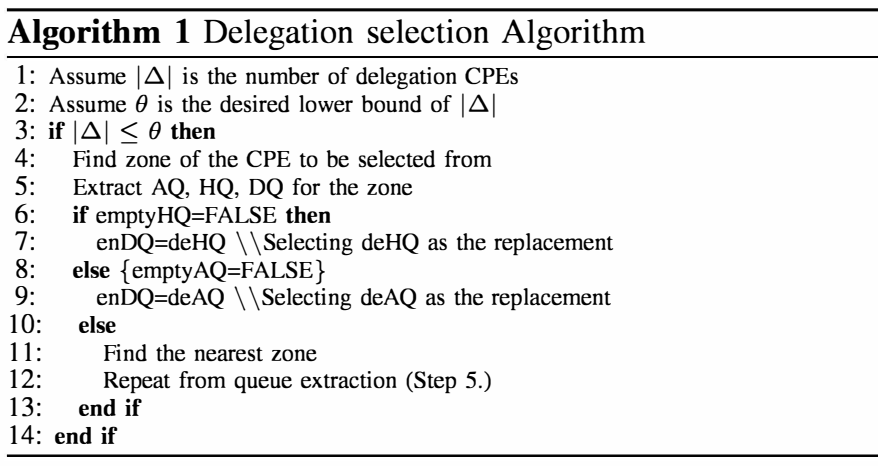

delegation representatives from different zones of the cell as more geographically dispersed delegated CPEs will help to create a more accurate Spectrum Map. Selection takes place after termination of data transmission (either voluntarily or forcefully) with the channels previously allocated for transmission now serving as sharing medium. If not selected for delegation, a CPE can relinquish its channels or hold onto the channels in anticipation of channel requirement in the near future. Holding time depends on the demand for the particular channels by other CPEs. However, such holding onto channels can result in that CPE being selected as a delegation member (using the same channels for sharing) if instructed by the BS. A delegated CPE continues to serve until it is instructed otherwise by the BS. This obligates the CPE to use the allocated channels only as means to share its spectrum usage with $\mathrm{BS}$ even if the CPE wants to go to the transmission state. The decision of keeping such a CPE in sharing state solely depends on the importance of that CPE in building the spectrum profile. Soon after the BS finds a suitable alternative, it allows the CPE to switch to the transmission state and use the same channel for data communication. Algorithm 1 describes the selection process of a delegation member.

In Fig. 1, we see how the coverage area of the BS is divided into zones. The BS attempts to find a delegated CPE for every zone. However, the BS might fail to do so for every zone.

\section{Intelligent beacon broadcast}

The IEEE 802.22 draft [1] mandates a BS to broadcast beacons as a part of advertising the availability of free channels to the CPEs. Instead of sending beacons in all the free channels, we adopt an intelligent beacon broadcasting technique where the beacons are sent only on selected channels depending on the requirement of idle CPEs. These selected channels are those that belong to the common set of the available channels for allocation and the set of channels which are estimated to be available for the idle CPEs.

If there are $K$ CPEs in a IEEE 802.22 cell and $p_{\text {alloc }}$ is the probability that a CPE is allocated at any instance then there are $\left(K p_{\text {alloc }}-|\Delta|\right)$ idle CPEs currently in the system. Let $\nu_{\text {est }}$ (where $\nu_{\text {est }} \in \hat{N}$ ) to be the estimated set of available channels for the idle CPEs with $\hat{N}$ being the total set of channels in the spectrum and $|\hat{N}|=N$. If $\gamma_{\text {free }}^{b}$ be the set of allocable set of channels on offer for the BS then beacons are send only on the channels belong to $\nu_{e s t} \cap \gamma_{\text {free }}^{b}$. We assume a single beacon is sent per channel. We assume that $\gamma_{\text {free }}^{c}$ is the set of free channels at a CPE $c$ and this contains 
the channels on which the CPE tunes its radio for beacon reception. Therefore, assuming negligible time for broadcast and propagation, probability of synchronization is given by:

$$
P(C P E \text { receiving beacon })=\frac{\left|\nu_{e s t} \cap \gamma_{\text {free }}^{b} \cap \gamma_{\text {free }}^{c}\right|}{\left|\nu_{\text {est }} \cap \gamma_{\text {free }}^{b}\right|\left|\gamma_{\text {free }}^{c}\right|}
$$

A beacon contains the IDs of all the channels on which the beacons are being sent. Therefore, a $\mathrm{CPE}, c$, once receiving a beacon sends back channel allocation requests in all the advertised channels along with the actual spectrum usage at $c \Upsilon^{c}=\left\{\varphi_{q}^{c} \mid \forall c h_{q} \in \hat{N}\right\}$. The completion of BS-CPE handshaking process is marked by the reception of the channel allocation request by BS in any of the $\left|\nu_{e s t} \cap \gamma_{\text {free }}^{b}\right|$ channels. Therefore,

$P($ Successful handshaking $)=\frac{\left|\nu_{\text {est }} \cap \gamma_{\text {free }}^{b} \cap \gamma_{\text {free }}^{c}\right|}{\left|\nu_{\text {est }} \cap \gamma_{\text {free }}^{b}\right|^{2}\left|\gamma_{\text {free }}^{c}\right|}$

This intelligent broadcasting scheme reduces the number of redundant beacons and increases the probability of synchronization with 0.67 in the best case and 0.35 in the worst case.

\section{INTERPOLATION OF SPECTRUM DATA}

Estimating the spectrum usage at any CPE from a given set delegation points is non-trivial. In essence, we seek to define a continuous two-dimensional interpolation function which passes through all the given irregularly-spaced data points ${ }^{1}$.

We have $|\Delta|$ cognitive radio enabled delegation CPEs and let the co-ordinates of the $i$ th delegation CPE $\delta_{i}$ be $\left(x_{i}, y_{i}\right)$. Also, this CPE records some data value of $z_{i}$. The data value can correspond to one of the many radio parameters like SNR, duty cycle, or detected energy for a particular channel that the CPE is sensing. Now, given $\delta$ such triplets $\left(x_{i}, y_{i}, z_{i}\right)$, we seek to find a two dimensional interpolation function $f(x, y)=z$ that will be continuous and differentiable, passing through all the data points i.e., $f\left(x_{i}, y_{i}\right)=z_{i}$, and should conform to real life values.

We use Shepard's [11] method of interpolation for irregularly spaced data points in a two dimensional region to estimate the spectrum usage at $c$. If $e_{q}^{i}$ is the value of the detected energy at $\delta_{i}$ for channel $c h_{q}$, then interpolated spectrum usage using Shepard's method [11] is given as:

$$
\Phi^{c}=\sum_{q=1}^{N} \phi_{q}^{c}=\sum_{q=1}^{N} \frac{\sum_{\delta_{i} \in R^{c}} w_{i}^{c} e_{q}^{i}}{\sum_{\delta_{i} \in R^{c}} w_{i}^{c}}
$$

The process of computing $\Phi^{c}$ is shown in Algorithm 2. Detailed discussion on the method of finding $\Phi^{c}$ is discussed in [11]. From the estimated spectrum usage at $c$, the BS finds the set $\psi_{\text {est }}^{c}$ (set of free channels at $c$ ) applying hypothesis analysis on estimated channel usages of all channels.

The complexity of algorithm 2 is $O\left(m^{2} N\right)$ where $N$ is the number of channels and $m$ is the number of delegation CPEs involved in the interpolation. Since the number of delegation CPEs is a constant, the average case complexity is $O(N)$.

${ }^{1}$ We use the delegation CPEs as the data gathering points, hence we also refer to them as 'data points'.

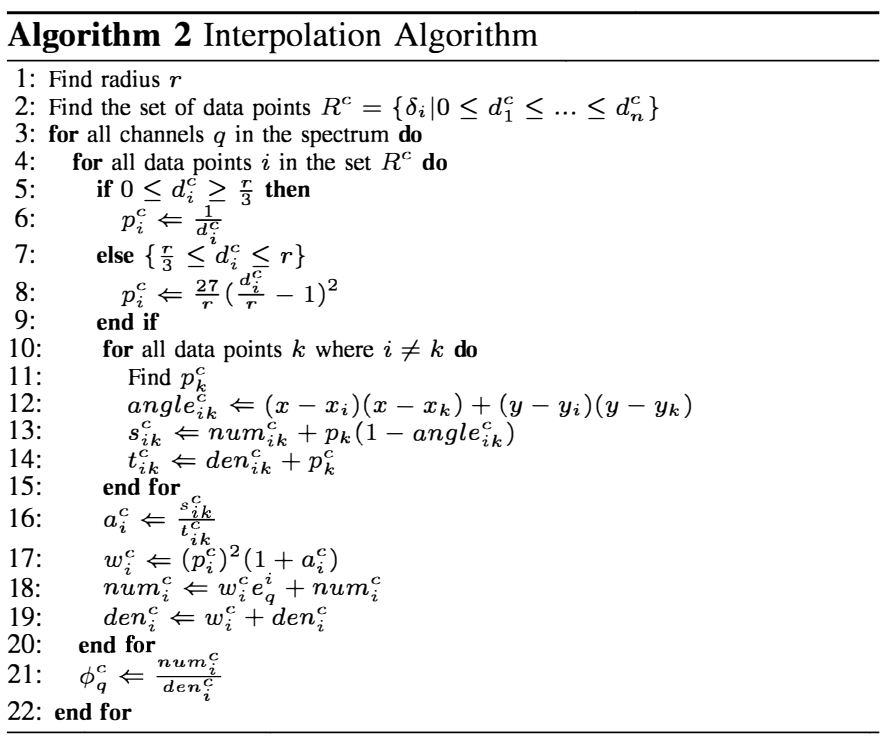

\section{Performance based Channel Allocation}

Successful beacon reception from a CPE initiates the BS to calculate performance metrics and allocate suitable channels to the CPE. Such metrics include channel capacity, spectrum efficiency, throughput, bit error rate etc.

\section{A. Performance metrics}

The interference experienced by any CPE is due to the primary transmitters as well as other transmitter-receiver pairs using the same channel. Let us suppose the respective interferences experienced by CPE $c$ located at $\left(x_{c}, y_{c}\right)$ in channel $c h_{q}$ are $\phi_{q}^{c}$ (estimated using eqn. 3) and $I_{q}^{c}=\sum_{\forall j \in \kappa^{q}} P\left|h_{q}^{j}\right|^{2}$ with $\kappa^{q}$ being the set of all other CPEs using channel $c h_{q}$.

Below we discuss BS calculating four of numerous possible performance metrics namely channel capacity, spectral efficiency, network throughput, and bit error rate.

Channel Capacity : The channel capacity $C_{q}^{c}$ for channel $c h_{q}$ is calculated using Shannon-Hartley theorem [10].

$$
C_{q}^{c}=B \log _{2}\left(1+\frac{P\left|h_{q}^{c}\right|^{2}}{\phi_{q}^{c}+I_{q}^{c}}\right)
$$

where $B$ is the channel bandwidth.

Secondary Network Throughput : If a BS transmits with power $P$ to $\mathrm{CPE} c$, then the transmission rate considering all other secondary communication is given by

$$
\pi_{q}^{c}=\log \left(1+\frac{P\left|h_{q}^{c}\right|^{2}}{I_{q}^{c}+\phi_{q}^{c}+\sigma^{2}}\right)
$$

where the received signals are corrupted by zero-mean additive white Gaussian noise of power $\sigma^{2}$. Network throughput for a particular channel is the sum the transmission rates of all the BS-CPE pairs using the same channel $c h_{q}$ and given as:

$$
\Pi_{q}=\sum_{\forall j \in \kappa^{q}} \pi_{q}^{c}=\sum_{\forall j \in \kappa^{q}} \log \left(1+\frac{P\left|h_{q}^{c}\right|^{2}}{I_{q}^{c}+\phi_{q}^{c}+\sigma^{2}}\right)
$$

Spectral Efficiency : Spectral efficiency provides an indication of how efficiently a bandwidth-limited frequency spectrum is used. Link spectral efficiency in bits $/ \mathrm{sec} / \mathrm{Hz}$ is defined as the net bit-rate that can be achieved by a link per 
channel bandwidth (Hz). Thus, link spectral efficiency for $c h_{q}$ between the BS and CPE $c$ is

$$
\xi_{q}^{c}=\frac{1}{B} \log \left(1+\frac{P\left|h_{q}^{c}\right|^{2}}{I_{q}^{c}+\phi_{q}^{c}}+\sigma^{2}\right)
$$

The system spectral efficiency also in bits/sec/Hz is defined as the maximum throughput, summed over all nodes, divided by the channel bandwidth. Therefore, system spectral efficiency is

$$
\Xi_{q}=\sum_{\forall j \in \kappa^{q}} \xi_{q}^{c}=\frac{1}{B} \sum_{\forall j \in \kappa^{q}} \log \left(1+\frac{P\left|h_{q}^{c}\right|^{2}}{I_{q}^{c}+\phi_{q}^{c}}+\sigma^{2}\right)
$$

Spectral efficiency gives the number of secondary nodes that can be simultaneously supported by the available spectrum in a geographic area. In other words, we regard it as a quantitative measure for self-coexistence of IEEE 802.22 networks.

Bit error rate : The bit error rate (BER) between BS and $\mathrm{CPE}$ is dependent on the modulation and coding schemes for communication. For DPSK, BER is given by [7]

$$
B E R_{q}^{D P S K}=\frac{1}{2} e^{-\frac{E_{b}}{\eta}}
$$

where $E_{b}$ is the average energy per bit transmitted and $\eta / 2$ is the noise power spectral density. The former is expressed as $E_{b}=P / \pi_{q}^{c}$. Noise power spectral density is given by $\eta=$ $\phi_{q}^{c} / B$. Substituting the values in equation (9), we get,

$$
B E R_{q}^{D P S K}=\frac{1}{2} e^{-\frac{P B}{\pi_{q}^{c} \phi_{q}^{c}}}
$$

If there are $Q$ bits in a transmitted packet then the packet error rate is given by $P E R=1-(1-B E R)^{Q}$. BER and PER can be calculated for any modulation scheme.

\section{B. Allocation process}

The allocation process by the BS is initiated by reception of channel allocation reply from any CPE $c$ in any of the $\nu_{e s t} \cap$ $\gamma_{\text {free }}^{b} \cap \gamma_{\text {free }}^{c}$ channels. Such allocation reply is accompanied with raw spectrum usage at $c, \Upsilon^{c}=\left\{\phi_{j}^{c} \mid \forall c h_{j} \in \hat{N}\right\}$. BS creates the set of available channels $\rho_{a v l}^{c}=\left\{j \mid \forall \phi_{j}^{c}<T\right\}$ allocable to $c$. Channels used by other allocated or delegated CPEs in the interference range of $c$ can not be allocated to $c$ for obvious reasons. In order to ensure this, the $\mathrm{BS}$ finds the $\mathrm{AQ}, \mathrm{DQ}$ and $\mathrm{HQ}$ of all the zones within the interference range of $c$. From the AQs, the BS creates the set $\widehat{A Q}^{c}$ with only those channels which are allocated to CPEs within the interference range of c. Similarly $\widehat{D Q}^{c}$ and $\widehat{H Q}^{c}$ are created. No channels in $\widehat{A Q}^{c}$ and $\widehat{D Q}^{c}$ can be allocated to avoid co-channel interference. BS updates the set of available channels for allocation $\rho_{a v l}^{c}$ excluding all such channels. For all the rest of the channels in $\rho_{a v l}^{c}$, expected channel performance (can be any desirable parameter or a combination of some) is calculated and given a score. $\rho_{a v l}^{c}$ is further updated with descending order of channel score. There is also the set $\widehat{H Q}^{c}$ whose channels are held by other idle CPEs in the interference range of $c$. The purpose of allowing the CPEs to hold onto channels is not making them go through the entire handshaking process and expedite channel allocation. Therefore, BS always tries to allocate the best channel among $\left|\rho_{a v l}^{c}\right|$ not belonging to $\widehat{H Q}^{c}$, if possible.

\section{Simulation Results}

We simulate TV stations in a $500 \times 500$ sq. miles grid as shown in Fig 3 using TV station location distribution in [8] [9]. The total number of channels considered is 50 with bandwidth of $6 \mathrm{MHz}$. The power-profile of the TV stations ranges between $10 \mathrm{~kW}-1 \mathrm{MW}$. We identify two regions with dense and sparse primary densities (mimicking big cities and small towns) and deploy IEEE 802.22 networks (WRAN cells) shown in Fig. 3. Locations of different types of nodes and transmission patterns are different in the two scenarios while total number of CPEs, density, and the number of delegated CPEs are kept the same. We evaluate the proposed technique both in terms of accuracy of spectrum usage estimation and performance of the allocation algorithm.

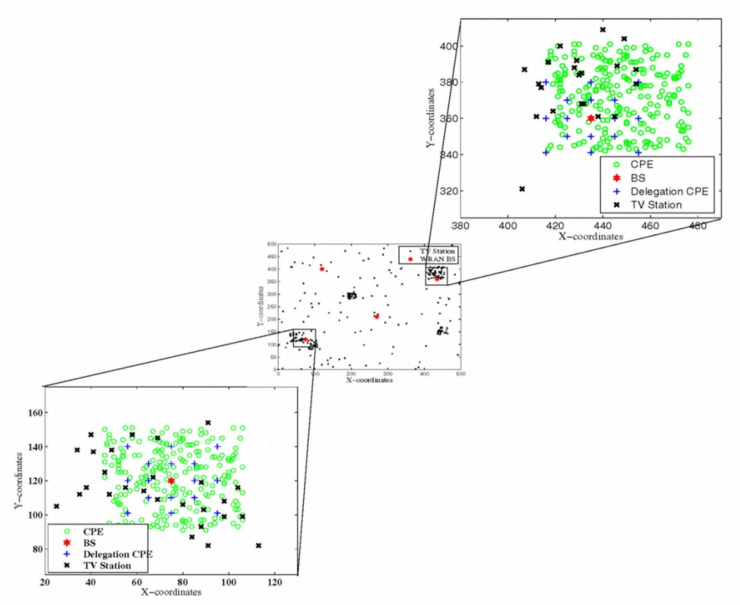

Fig. 3. Simulation grid with primary transmitters, secondary BSs and CPEs

In figures 2(a), 2(b), and 2(c), 2(d), we show the estimated and actual signal strengths for two regions. The figures reflect the success of the interpolation technique to capture the nature of spectrum usage in the regions. By comparing the estimated and actual signal strength values for both region, we get the error in estimation of the order $10^{-6} \mathrm{~mW}$ which is too small to alter the occupancy decision vector.

Fig. 4 shows the channel occupancy vectors of two different CPEs located in two regions. In both the cases the prediction of channel occupancy is highly accurate with only 6 and 3 false positives respectively. It is important to note that none of these variations are false negatives which is essential for secondary communication. Our results show that on an average the probability of false positive is as low as 0.08 . Such accurate estimation can lead upto $92 \%$ reduction in beacon broadcast.

Figs. 5(a) and 5(b) show the spatial distribution of channel capacity for the two scenarios. Such spatial distribution helps the BS not only to find the best candidate channel for a CPE but also the best candidate CPE for a particular channel which will realize the highest achievable capacity for that channel. The bandwidth of the channels are kept constant at $1 \mathrm{MHz}$.

Fig. 6(a) shows the performance of allocation method for different probability of white space. We show the results for worst case scenario when upto 200 simultaneous idle CPEs want to get allocated. The scheme performs very well for realistic simultaneous allocable CPEs with realistic probability of white space (0.6 and higher.).

Utilization of free channels using our scheme is shown in Fig. 6(b). We see that on an average more than $50 \%$ of the 


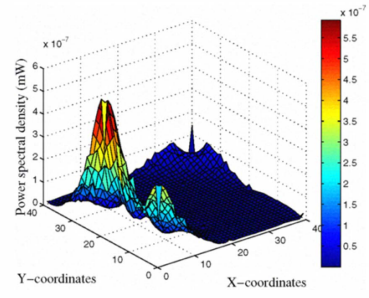

(a)

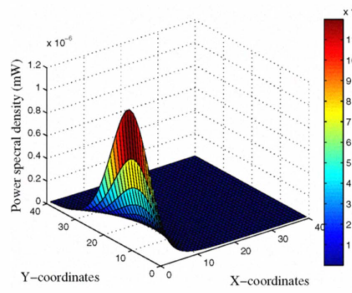

(b)

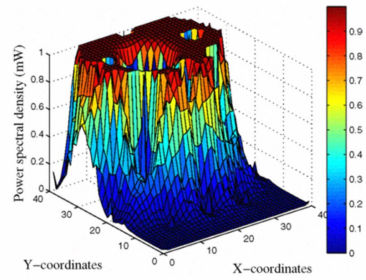

(c)

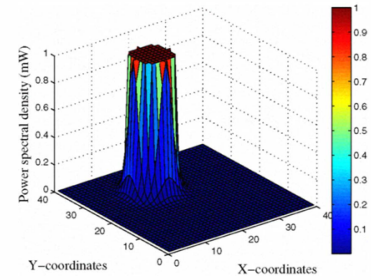

(d)

Fig. 2. (a) (b) Estimated and actual signal strengths of a channel for Region 1; (c) (d) Estimated and actual signal strengths of a channel for Region 2

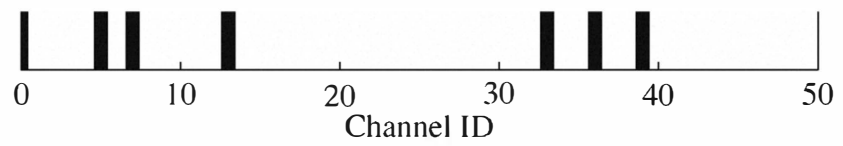

(a) Estimated channel occupancy of CPE \#38 in Region 1

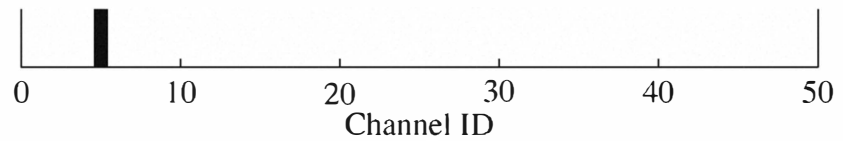

(a) Actual channel occupancy of CPE \#38 in Region 1

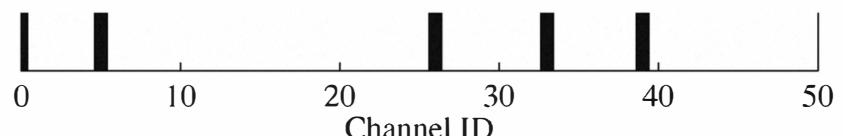

(a) Estimated channel occupancy of CPE \#106 in Region 2

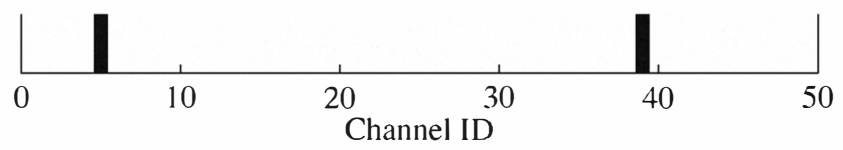

(b) Actual channel occupancy of CPE \#106 in Region 2

Fig. 4. Estimated and actual channel occupancy of two CPEs in two regions

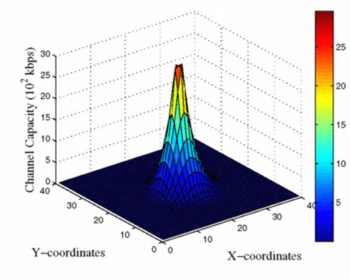

(a)

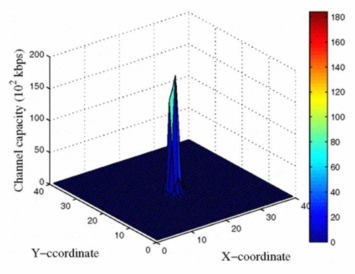

(b)
Fig. 5. Spatial distribution of channel capacity for two regions

free channels of the spectrum are being utilized. Utilization increases with increasing demand from idle CPEs.

\section{CONCLUSIONS}

In this paper, we proposed a spectrum usage estimation technique for IEEE 802.22 networks. We used Shepard's interpolation method to construct spectrum map. We showed how the data gathered only from the delegated CPEs can be used to evaluate the channel occupancy vector which is then used to communicate with the idle CPEs. We also proposed a channel allocation algorithm which enhances channel and network

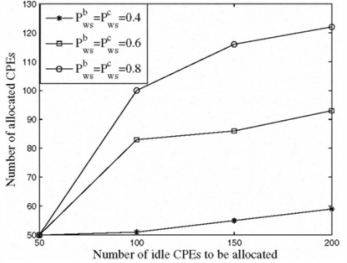

(a) Allocation for different probability of white space

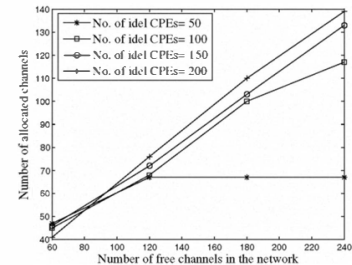

(b) Allocation for differnt number of idle CPEs
Fig. 6. Efficiency of channel allocation technique

performance. Through simulation experiments, we showed the accuracy of the spectrum estimation and effectiveness of the allocation technique.

\section{REFERENCES}

[1] IEEE P802.22/D1.0 Draft Standard for Wireless Regional Area Networks Part 22: Cognitive Wireless RAN Medium Access Control (MAC) and Physical Layer (PHY) Specifications: Policies and Procedures for Operation in the TV Bands, April 2008

[2] IEEE 802.22, Working Group on Wireless Regional Area Networks (WRAN), http://grouper.ieee.org/ groups /802/22

[3] Akyildiz, I. F.; Lee, W. Y.; Vuran, M.C.; Mohanty, S.; , "NeXt Generation/Dynamic Spectrum Access/Cognitive RadioWireless Networks: A Survey, " Elseview Journal of Computer Networks, Vol. 50, pp. 2127-2159, September 2006

[4] Hou-Shin Chen; Wen Gao; Daut, D.G.; , "Signature Based Spectrum Sensing Algorithms for IEEE 802.22 WRAN," Communications, 2007. ICC '07. IEEE International Conference on , pp. 6487-6492, 24-28 June 2007

[5] Ganesan, G.; Li Ye; , "Cooperative Spectrum Sensing in Cognitive Radio, Part II: Multiuser Networks," Wireless Communications, IEEE Transactions on , vol.6, no.6, pp.2214-2222, June 2007

[6] Lazos, L.; Sisi Liu; Krunz, M.; , "Spectrum Opportunity-Based Control Channel Assignment in Cognitive Radio Networks," Sensor, Mesh and Ad Hoc Communications and Networks, 2009. SECON '09. 6th Annual IEEE Communications Society Conference on , pp. 135-143, 22-26 June 2009

[7] Proakis, J. G.; , Digital Communications, 4th ed. McGraw-Hill, 2001

[8] Riihijarvi, J.; Mahonen, P.; Wellens, M.; Gordziel, M.; , "Characterization and modelling of spectrum for dynamic spectrum access with spatial statistics and random fields," Personal, Indoor and Mobile Radio Communications, 2008. PIMRC 2008. IEEE 19th International Symposium on , pp.1-6, 15-18 Sept. 2008

[9] Riihijarvi, J.; Mahonen, P.; "Exploiting Spatial Statistics of Primary and Secondary Users towards Improved Cognitive Radio Networks," Cognitive Radio Oriented Wireless Networks and Communications, 2008. CrownCom 2008. 3rd International Conference on , pp.1-7, 15-17 May 2008

[10] Shannon, C. E.; , "A mathematical theory of communication," Bell Syst. Tech. J., vol. 27, pp. 379-423, 623-656, 1948

[11] Shepard, D.; , "A Two-Dimensional Interpolation Function for Irregularly-Spaced Data," ACM National Conference, 1968

[12] Sengupta, S.; Chandramouli, R.; Brahma, S.; Chatterjee, M.; , "A game theoretic framework for distributed self-coexistence among IEEE 802.22 networks," Global Telecommunications Conference, 2008. IEEE GLOBECOM 2008. IEEE , pp. 1-6, Nov. 30 2008-Dec. 42008

[13] Tachwali, Y.; Barnes, W.J.; Basma, F.; Refai, H.H.; , "The Feasibility of a Fast Fourier Sampling Technique for Wireless Microphone Detection in IEEE 802.22 Air Interface," INFOCOM IEEE Conference on Computer Communications Workshops , 2010 , pp. 1-5, 15-19 March 2010 